\title{
BMJ Open 'Do Well B.': Design Of WELL Being monitoring systems. A study protocol for the application in autism
}

To cite: Dutheil F, Chambres $P$, Hufnagel $C$, et al. 'Do Well B.': Design of WELL Being monitoring systems. A study protocol for the application in autism. BMJ Open 2015;5:e007716. doi:10.1136/bmjopen-2015007716

- Prepublication history for this paper is available online. To view these files please visit the journal online (http://dx.doi.org/10.1136/ bmjopen-2015-007716).

Received 18 January 2015 Accepted 21 January 2015

CrossMark

For numbered affiliations see end of article.

Correspondence to Dr Frédéric Dutheil; frederic.dutheil@acu.edu.au

\section{ABSTRACT}

Introduction: Individuals with autism spectrum disorder (ASD) have difficulties in communication and social interaction resulting from atypical perceptual and cognitive information processing, leading to an accumulation of anxiety. Extreme overloading experienced internally may not be externally visible. Identifying stressful situations at an early stage may avoid socially problematic behaviour from occurring, such as self-injurious behaviour. Activation of the autonomous nervous system (ANS) is involved in the response to anxiety, which can be measured through heart rate variability and skin conductance with the use of portable devices, non-intrusively and pain-free.

Thus, developing innovative analysis of signal perception and reaction is necessary, mainly for non-communicative individuals with autism.

Methods and analysis: The protocol will take place in real life (home and social environments). We aim to associate modifications of the ANS with external events that will be recorded in a synchronous manner through a specific design (spy glasses with video/ audio recording). Four phases will be carried out on ASD participants and aged-matched controls:

(1) 24-hour baseline pre-experiment (physical activity, sleep), (2) $2 \mathrm{~h}$ in a real life situation,

(3) $30 \mathrm{~min}$ in a quiet environment, interrupted by a few seconds of stressful sound, (4) an interview to record feelings about events triggering anxiety. ASD and control participants will be together for phases 2 and 3 , revealing different physiological responses to the same situations, and thus identifying potentially problematic events. The novelty will be to apply time-series analyses (which led to several Nobel Prizes in quantitative finance) on ANS series (heart rate, heart rate variability, skin conductance) and wrist motion.

Ethics and dissemination: Ethical approval has been obtained from Ethics Committee of ClermontFerrand (South-East I), France (2014-A00611-46). Trial findings will be disseminated via open-access peer-reviewed publications, conferences, clinical networks, public lectures and our websites.

Trial registration number: ClinicalTrials identifier NCT02275455.
Strengths and limitations of this study

- 'Do Well B.' is a multidisciplinary project that builds innovative links between autism spectrum disorders (ASD), audiovisual signal processing, physiological response, and stochastic modelling and statistics.

- This study investigates the novel concept of advancing a personal health system (PHS) for early detection of challenging behaviours for ASD individuals. Such a PHS could be easily and daily used, outside the hospital. Moreover, early comprehensive and targeted behavioural interventions could improve social communication and reduce anxiety and aggression.

- We limited our project to modelling, experiments and data processing. The deliverable objective is numerical codes for early detection of autism disorders. This may appear as a limitation, yet our project seems reachable.

- Finally, we will disentangle different behaviours such as anxiety, rest and physical activity. Thus, this study has a huge potential in the field of 'mobile e-Health solutions'.

\section{BACKGROUND}

Autism spectrum disorder (ASD) is a set of heterogeneous neurodevelopmental conditions characterised by early-onset difficulties in social interaction and communication, with unusually restricted, repetitive behaviour and interests. ${ }^{1}$ Constantly increasing, the worldwide prevalence is reaching $1 \% .^{1}{ }^{2}$ Even if genetics has a key role in its aetiology, environmental factors in early development are also influential. ${ }^{3}$

Atypical neural development ${ }^{4}$ commonly underpins atypical cognitive profiles, such as impaired social cognition and social perception, executive dysfunction, and atypical perceptual and information processing. ${ }^{5}$ Most individuals with autism suffer from psychological comorbidities such as social anxiety, 
attention-deficit and oppositional defiant disorders. ${ }^{6}$ Social adapting behaviours decrease as symptoms due to a dysfunction in sensory processing increase, through the increase of anxiety. ${ }^{7}$ Individuals with autism often experience states of emotional or cognitive overload. ${ }^{8}$ Measurements taken from home and school environments have shown that experiences of extreme overloading internally may not be visible externally. For example, an individual with ASD can have a resting heart rate (HR) twice the level of non-autistic peers, while outwardly appearing calm and relaxed. ${ }^{8}$ Thus, tools to measure internal anxiety are needed.

Activation of the autonomous nervous system (ANS) is involved in response to stress and anxiety. ${ }^{9}$ The ANS is a balance between sympathetic and parasympathetic activity. When an individual is threatened, parasympathetic activity is inhibited, triggering a series of responses to promote survival (ie, increased HR and increased blood flow to the limbs). By contrast, when the surrounding environment is perceived as safe, the parasympathetic system is activated, slowing the HR, promoting social behaviour and homeostatic functions. ${ }^{10}$ Analysing ANS variations is important for understanding and helping people with ASD because ANS activation influences and is influenced by sensory information, social interactions and physical activity. ${ }^{8}$ The most common measures of ANS are HR variability (HRV) and skin conductance (SC).

The balance between sympathetic and parasympathetic activity can be assessed by measuring HRV. ${ }^{11}$ Low HRV has been associated with anxiety ${ }^{12}$ and stress, ${ }^{9}$ and HRV may provide a good tool to infer emotions. ${ }^{10}$ Moreover, individuals with autism exhibit less cardiac capacity to adjust for demanding tasks, ${ }^{13}$ and also have reduced cardiac parasympathetic activity. ${ }^{14}$ Conveniently, HRV can be measured non-intrusively and pain-free.

The sympathetic nervous system, influenced by changes in emotions, releases acetylcholine, which acts on muscarine receptors, causing a subsequent burst of sweat and increased SC. ${ }^{15}$ For individuals with ASD, SC is augmented with stress, ${ }^{16}$ the 'fight or flight' response activation, ${ }^{8}$ and is also related to cognitive arousal. ${ }^{17}$ Similar to HRV, SC can be measured with the use of a portable device, non-intrusively and pain-free. Moreover, SC reacts immediately and is not influenced by haemodynamic variability or neuromuscular blockade. ${ }^{15}$

Early comprehensive and targeted behavioural interventions can improve social communication and reduce anxiety and aggression. ${ }^{18} 19$ For individuals with ASD, identifying stressful situations at an early stage may avoid the occurrence of socially problematic behaviour, such as self-injury. Most individuals with ASD suffer from abnormal sensitivity experiences, ${ }^{20}$ particularly within the acoustic environment, ${ }^{521}$ which may cause accumulation of anxiety. Thus, developing innovative analysis of signal perception and reaction is necessary, ${ }^{22}$ mainly for non-communicative people with autism.

The primary hypothesis is that variations of HR timeseries will allow fast detection of anxiety before an extreme overloading is experienced internally, even if this overloading is yet to be visible externally. Moreover, HR and HRV time-series will also detect an absence of an expected reaction to a warning situation such as crossing at the traffic lights.

The secondary hypothesis is that modifications of the parameters of the SC time-series will also detect anxiety before an extreme overloading; HR-series and SC-series will detect different responses and may be complementary, as will other physiological responses such as wrist motion or respiratory rate. We will use statistics for multivariate physiological time-series (HR, HRV, SC, wrist motion, respiratory rate). HR responses, measured with the use of an HR thin elasticised transmitter belt placed on the chest, will be sufficient to compare with a Holter ECG. Respiration rate, measured with the same HR transmitter belt, will be helpful to detect anxiety. Reactions of different body segments measured with an accelerometer will also be helpful to distinguish anxiety from physical activity.

Therefore, our aim will be to link the results from analysis of physiological time-series to environmental signals to build a personal health system (PHS) for early detection of challenging behaviour in individuals with autism in real life situations in a community setting.

\section{METHODS}

\section{Trial design}

We aimed to associate modifications of ANS (variations of the HR and SC time-series) with external events. As previously stated, individuals with ASD encounter difficulties in social interactions and would also experience challenges following any laboratory-based protocol. Subsequently, we decided to conduct a study in real life situations, outside the hospital or laboratory, in a so-called ecological situation. Statistical calculations are based on time-series analysis. First, we detect times of change for the mean of HR series and high-frequency $(\mathrm{HF})$ as well as low-frequency (LF) energies. By doing so, we obtain segments where the different physiological series stay in a steady state. Next, we compute the mean HR, HF and LF energies of HRV on each segment. Then, these data are used for classifications to qualify the thresholds of stress for individuals (see details in section on statistics below). ${ }^{23}$

The study design permits the synchronous recording of data from HR, SC, respiratory rate, wrist motion and the contextual events. Responses to four phases will be conducted during this research on ASD participants and aged-matched controls: (1) $24 \mathrm{~h}$ pre-experimental recording for baseline, (2) a $2 \mathrm{~h}$ experimental recording in real life situations, (3) a 30 min recording in a quiet environment, interrupted by a few seconds of stressful sound, (4) within the realms of possibility, an interview will be achieved with the participant to capture comments and feelings about events triggering anxiety. Each participant (ASD and control) will undergo phases 1 to 4 , 
with the recording of data from HR, SC and contextual events during phases 1 to 3 . ASD participants will be paired with their matched control during phases 2 and 3 . Thus, they will live the same situation at the same time, allowing us to detect different responses between them and identify potentially stressful situations for ASD participants.

\section{Participants}

Inclusion criteria for participants with autism are:

- A reliable diagnosis of ASD, based on the autism diagnostic interview-revised (ADI-R), ${ }^{24}$ and the autism diagnostic observation schedule-generic (ADOS-G) ${ }^{25}$ The Vineland adaptive behavior scales $(\text { VABS })^{26}$ will measure personal and social skills of participants.

- No change in medical prescription in the previous 3 months and during the experiment.

- Age between 10 and 25 years because, on the one hand, we need to examine participants in a moderately free situation and this will be more acceptable for parents if the children are 10 or above; and on the other hand, an absolutely reliable diagnosis of ASD (established with ADI-R and ADOS international tests) is hard to reach in those individuals aged over 25 years.

The same inclusion criteria will apply for the paired individuals with the exception of ASD. The paired control will be chosen among the friends of the individual with ASD. In cases of difficulties recruiting controls, the psychologist adviser may serve as control.

Recruitment and study protocol will start on February 2015 and can continue to December 2015.

\section{Outcomes}

The primary objective will be to demonstrate that analysis of HR and HRV time-series obtained from a Holter ECG recording will detect anxiety before an extreme overloading experienced internally becomes visible externally (figure 1).

Secondary objectives will be to demonstrate that:

- SC will detect anxiety before an extreme overloading becomes visibly apparent;

- HRV and SC will detect different types of responses but can also provide complementary information;
- HRV and SG will also detect the absence of reaction to a warning situation, such as crossing at the traffic lights;

- HRV measured with the use of an HR transmitter belt placed on the chest will be sufficient compared with a Holter ECG;

- Respiration rate measured with the use of an HR transmitter belt placed on the chest will be helpful in detecting anxiety;

- Movement of the body measured with an accelerometer worn at the wrist will also be helpful in detecting anxiety.

\section{Intervention}

A direct 'medical' environment (psychiatrist, psychological adviser, general practitioner or other significant individual involved with the ASD participant) will be included in this study and will have to agree to the participation of the individual with ASD in our study.

In order to list the potentially problematic sounds and situations, a $1 \mathrm{~h}$ interview will be conducted with parents and, if possible, with efficient speaking participants. To understand more about anxiety in their environment, questions will be asked about individuals' stressful situations and sounds, as well as related health issues such as hearing capacity, medications, sleeping habits and personal interests.

More precisely, the four phases will consist of (table 1):

- Phase 1: A $24 \mathrm{~h}$ phase will be used to record HR and SC during sleep to record baseline data. In addition, a short period of $2 \mathrm{~min}$ of physical activity (riding a stationary bicycle, or running or jumping) will be used to check the sensitivity of HR responses as HR should respond linearly to exercise stimuli, either during resistance ${ }^{27}$ or endurance ${ }^{28}$ activity.

- Phase 2: Participants with ASD and their paired counterpart will be exposed to a 'real life situation' over a period of $2 \mathrm{~h}$. Both will be exposed to the same situation simultaneously. This should reveal different physiological responses (HR and SC data) to identical situations, and to identify which sound events and situations are potentially problematic. The 'real life situation' will start with a 5 min baseline rest (ie, sitting quietly with a familiar person) in order to later
Figure 1 In autism spectrum disorders, accumulation of anxiety experienced internally may not be visible externally, leading to socially problematic behaviour. Detecting anxiety at an early stage through analysis of physiological signals, before extreme anxiety overloading, may avoid the occurrence of socially problematic behaviour.

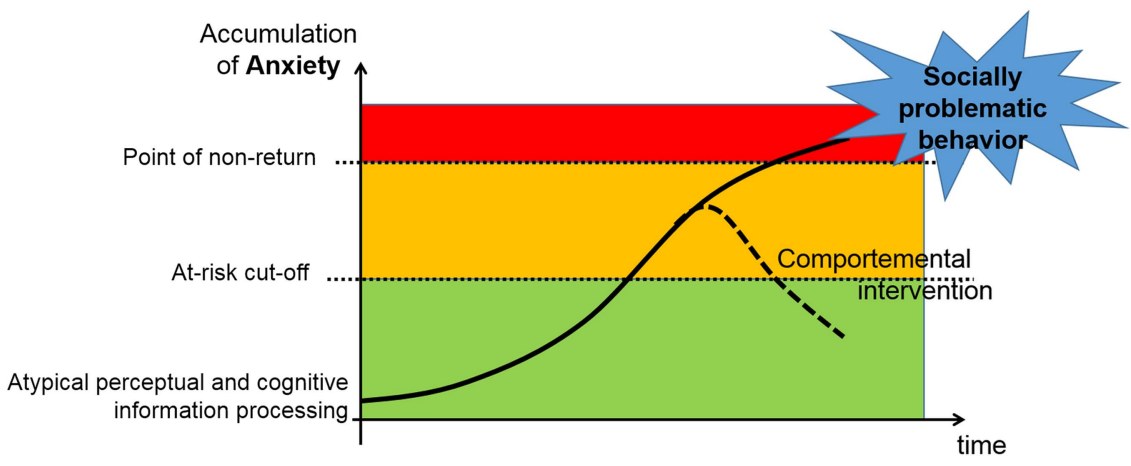


Table 1 Trial design

Participants with autism $(\mathrm{n}=30)$

Age-matched controls $(n=30)$

A $1 \mathrm{~h}$ interview to characterise autism and potentially stressful events

Phase 1 A $24 \mathrm{~h}$ pre-experimental recording for baseline (sleep, physical activity)

Phase 2 A 2 h experimental recording in a real life situation

Phase 315 min recording in a quiet environment, followed by a few seconds of stressful sound, followed by $15 \mathrm{~min}$ recording in a quiet environment

A $24 \mathrm{~h}$ pre-experimental recording for baseline (sleep, physical activity)

A $2 \mathrm{~h}$ experimental recording in a real life situation 15 min recording in a quiet environment, followed by a few seconds of stressful sound, followed by $15 \mathrm{~min}$ recording in a quiet environment

Phase 4 Interview to get comments on feelings about events that triggered anxiety

Interview to get comments on feelings about events that triggered anxiety

In yellow. During the $2 \mathrm{~h}$ experimental recording in real life and the 30 min recording in a quiet environment interrupted by a few seconds of stressful sound, the participant with autism and the age-matched control will be together, revealing different physiological responses to the same situations.

interpret physiological reactivity, specifically HRV and SC.

- Phase 3: ASD participants will be tested in a 'sound stressing situation', that is, a situation where a 'problematic' sound will be produced after spending $15 \mathrm{~min}$ in a very quiet situation (at home when possible). For very obvious ethical reasons, the problematic sound will be chosen among those that are weakly stressful and easily produced in the family environment (such as vacuum cleaner, doorbell or a specific voice) for a standard period. Directly after the sound exposure, physiological responses will be recorded for another 15 min quiet period.

- Phase 4: At least for controls and high-functioning ASD participants, a postexperimental interview will be conducted, in which comments and feelings can be expressed about events that triggered specific HR or SC modifications. To make this phase easy (especially regarding recollection of the psychological states), participants will be invited to watch some parts of the video and audio recording of the event. Data from this phase will be particularly useful to focus on physiological responses recorded during 'specific' events.

- The entire protocol from the neuropsychological evaluation to the end of phase 4 will be completed within 7 days.

\section{Material}

\section{Heart rate variability}

A Holter ECG and an HR transmitter belt will both measure HR data during phases 1 to 3 :

- A two-lead cardiscope ONE electrocardiogram Holter (Hasimed, Graz, Austria) with $24 \mathrm{~h}$ recording time, a $1024 \mathrm{~Hz}$ sampling rate and $1 \mu \mathrm{V}$ amplitude resolution, will be used.

- The Zephyr BioHarness BT (Zephyr Technology, Annapolis, USA), a thin elasticised HR transmitter belt placed on the chest, with $26 \mathrm{~h}$ recording memory, will be able to detect HRs between a range of 25 and $240 \mathrm{bpm}$, and respiratory rate within 3-70 breaths/min range. The aim will be to demonstrate that this relative inexpensive and easy-to-use device is sufficient to detect anxiety. The respiratory rate could also be a useful tool to detect anxiety. In addition, the Zephyr BioHarness BT also has the capacity to estimate the trunk's movements with a triaxial accelerometer.

The HR data will first be examined according to the recommendations of the European Society of Cardiology and the North American Society (Task Force). ${ }^{29}{ }^{30} \mathrm{HR}$ data will be explored simultaneously in time and frequency domains. ${ }^{31}$ The methodology developed by our team will also be applied. ${ }^{23}$

\section{SC and motion series}

SC will be measured in micro Siemens with sampling rates at 2, 4, 8, 16 and $32 \mathrm{~Hz}$ during phases 1 to 3 . The SC sensor (Q-Sensor-Affectiva, Massachusetts Institute of Technology, USA) is set on a wristband and has a $24 \mathrm{~h}$ battery life when logging. In addition, it will measure wrist movements with a built in triaxial accelerometer.

\section{Recording contextual events}

During phase 2, a set of 'spy glasses' (Active Media Concept, Paris, France) will be used to record simultaneously the physical and social environment from the participant's point of view. A camera and a microphone compose the 'spy glasses'. The photodetector is a Complementary Metal Oxide Semiconductor (CMOS) sensor $1 / 18$ with $720 \times 480$ resolution, a view angle of $60^{\circ}$, and records at 30 frames per second. Data are stored on a $32 \mathrm{~GB}$ MicroSDHC card allowing for $2 \mathrm{~h} 15 \mathrm{~min}$ of recording time. For participants who already wear corrective glasses, the camera and microphone will be placed on their own glasses.

\section{Statistics}

General design

Experiments will be conducted with two paired participants: the person with ASD and his age-matched 
control. Our aim is to measure the difference in regulation of physiological responses between the two participants, in order to build a PHS for the early detection of challenging behaviour in ASD individuals outside the hospital. We will process multivariate physiological timeseries (HR, HRV, SC, wrist motion, respiratory rate) in order to build a stress index. We will first use time-series segmentation in order to identify the segment boundary points in each univariate time-series. Second, we will compute the dynamical parameters associated with each segment, that is, mean value, HF and LF energies, and also fractal index. Third, we will use classification algorithms on the parameters obtained in the second step in order to obtain different classes corresponding to different levels of stress. Eventually, we will obtain, for each time, the level of stress, and will be able to compare it across the environmental conditions.

\section{Analyses for the primary outcome}

From heartbeat data, we will compute three time-series: RR-intervals (distance between two consecutive R-waves on ECG), HF and LF energies. HF and LF energies will be assessed with the use of wavelets that are strategically localised in time and frequency, ${ }^{32}$ contrary to Fourier analysis, which is not localised in time. Then, detection of abrupt changes will allow segmentation of the three time-series into shorter time series of a few minutes with a constant mean of RR-intervals, and HF and LF energies, respectively. ${ }^{23}$ 33-35 Moreover, we will also estimate the persistence or antipersistence of RR-intervals through a piecewise constant fractal index ('the Hurst index' $)^{36}{ }^{37}$ combining increment ratio statistics ${ }^{38}$ and detection of abrupt changes. ${ }^{35}$ Eventually, after having performed a Principal Component Analysis, ${ }^{39}$ we will disentangle clusters of variables characterising different behaviours of RR-intervals series such as anxiety, rest and physical activity. ${ }^{40}$

\section{Number of participants needed}

Stress effects on HR or HRV are documented in the literature. ${ }^{9}$ For instance, stress resulting from computer work induces a bilateral variation of $\mathrm{HR}$ of a magnitude greater than $5 \mathrm{bpm}$, without a significant change on LF energy, but a significant variation of HF energy. Specifically, Hjortskov et $a l^{41}$ demonstrated that, during stress periods, the $\log (\mathrm{HF})$ was 7.03 , while at rest, the $\log (\mathrm{HF})$ was 7.49 . This variation is plainly confirmed by others, ${ }^{29}$ who have shown that during night time, the $\log (\mathrm{HF}$ ) was $5.8 \pm 0.1$ (average value $\pm \mathrm{SD}$ ), or HR was 60.8 $\pm 1.3 \mathrm{bpm}$, and during daytime the $\log (\mathrm{HF})$ was $5.2 \pm 0.2$, or HR was $78.3 \pm 2.5 \mathrm{bpm}$. These data allowed us to compute the sample size for a unilateral test at level $\alpha=0.05$ for a change of $\log (\mathrm{HF})$ of approximately $0.3 \mathrm{log}$ $\mathrm{ms}^{2}$, meaning that we reach a power of 0.90 as soon as the sample size exceeds 5 , and for a change of $5 \mathrm{bpm}$ we also reach a power 0.90 as soon as the sample size exceeds 5. Eventually, even if we can process each single series as a time-series, ${ }^{23}$ we still need the occurrence of an overloading experience, which can be estimated at $\mathrm{p}=0.8$ during phase 2 . Thus, with a sample size of 15 , the probability of $\mathrm{k}$ overloading experience is given by the binomial law $\mathrm{B}(\mathrm{n}, \mathrm{p})$, and we get $\mathrm{P}(\mathrm{k}>6)=0.996$. More precisely, for $\mathrm{k}=7$, we get a power 0.95 . We will enrol 30 ASD participants and 30 age-matched controls to have a comfortable data set, allowing a huge rate of drop-outs.

\section{Analyses for the secondary outcomes}

We will mainly apply the same statistical treatment for the analyses of SC-series, respiratory rate and body motion. For instance, in a preliminary study, we have found that the SC for a 15-year-old participant with ASD in stressful conditions was greater than in normal resting conditions $(\mathrm{SC}=3.65 \pm 0.15 \mu \mathrm{S}$ vs $\mathrm{SC}=3.31 \pm 0.13 \mu \mathrm{S}$, respectively). So, at level $\alpha=0.05$, for a change of SC of size greater than $0.30 \mu \mathrm{S}$ and a sample size $\mathrm{k}=7$, we still reach a power 0.90 .

\section{Method taking into account missing, unused or invalid data} On each physiological series (HR, SC, respiratory rate, motion), missing or aberrant data are common and will be removed with the use of the preprocessing method. ${ }^{23}$ Then, data sets with more than $10 \%$ of aberrant or missing data will be rejected. ${ }^{29} 30$

\section{Measures to reduce or avoid bias}

Participant de-identification will blind assessors of participant status when processing data. Participants with autism will be paired with an age-matched control. The ANS will be measured through different devices.

\section{Ethics and dissemination}

'Do Well B.' is a multidisciplinary project. It involves eminent specialists in each field: probabilists and statisticians, autism scientists and neuroscientists, physiologists and cardiologists, and audio and audio-visual signal processing specialists. Implementation and conduct of the study will be monitored by the project management committee (authors) who have extensive experience in research and conducting clinical trials in ASDs, mathematics and engineering. Trial findings will be disseminated via open-access peer-reviewed publications, conferences, clinical networks, public lectures and our websites. The trial findings will also be made available to participants. Both collectively and for the participants, identifying stressful situations at an early stage may avoid socially problematic behaviour from occurring, and may permit early comprehensive and targeted behavioural interventions. We may determine unknown stressful events and, thus, target appropriate therapies. For the participants, our intervention may permit self-evaluation, giving them the chance to better anticipate and adapt to stressful situation on their own. This protocol may also incidentally detect cardiac disorders. Such an event will be communicated to the caregivers or the participant himself in the realm of possibility. Any abnormality discovered will not be covered by promoter's insurance (our 
observational study cannot lead to such an event) and will be supported by health insurance.

\section{DISCUSSION}

The main aim of "Design of well-being monitoring systems (Do Well B.)" (figure 2) is to use statistics of multivariate physiological time-series to build PHSs for early detection of challenging behaviour for ASD individuals, outside the hospital. Early comprehensive and targeted behavioural interventions can improve social communication and reduce anxiety and risk of aggression.

The idea is simple and corresponds to useful and awaited applications. Innovation lies in the potential for early detection of anxiety in autism disorders, that is, psychological health rather than physiological health. As pointed out by the WHO: "Health is a state of complete physical, mental, and social well-being and not merely the absence of disease or infirmity." We have observed huge advances in biological, physiological and psychological data processing inside the hospital. However, processing physiological signals outside the hospital is still in its early stages. So, the connection between health information and communication technology addresses a new challenge. Outside the hospital, in so-called ecological situations, we cannot use heavy devices such as functional MRI. However, today's technology allows the continuous monitoring of physiological signals with embedded devices (HR, SC, motion), so-called physiological time-series. Time-series analysis has a long history in econometrics and quantitative finance, which has led to several Nobel prizes being won, ${ }^{42-45}$ but a shorter history in health and medicine. Thus our challenge is to deduce modification of psychological regulation from the variation of such physiological signals. In ecological situations, there is feedback from the environment that can manifest in audio signals and audiovisual cues that result in a physiological answer. Audio and audiovisual signals are both continuous time random signals and should be studied by stochastic modelling and ad hoc

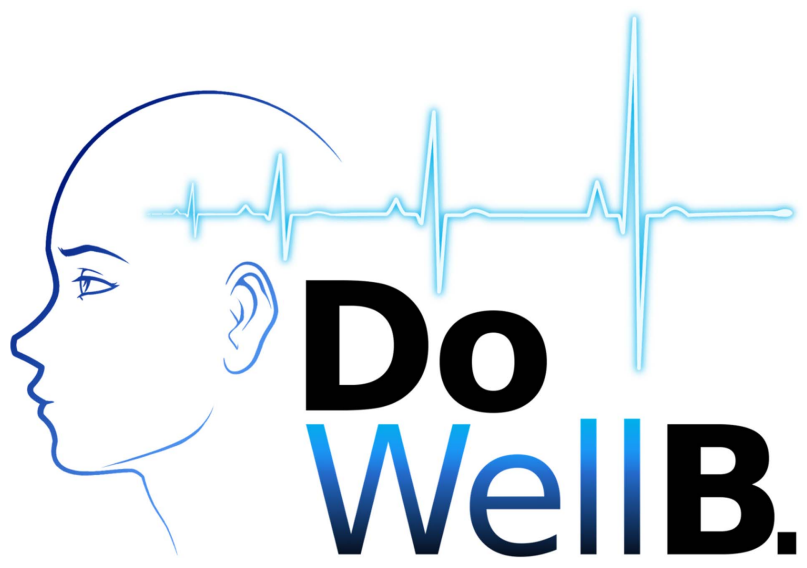

Figure 2 Design Of WELL Being monitoring systems (Do Well B.): from signal perception to emotional reaction. statistics tools. We will use and improve stochastic modelling and statistics of physiological and environmental signals. 'Do Well B.' is a multidisciplinary project that builds innovative links between ASD, audiovisual signal processing, physiological response, and stochastic modelling and statistics. The use of "mobile e-Health solutions for disease management, based on multi-parametric data including physiological measurements" is addressed in the Information and Communication Technology Work Program of the European Community.

\section{Potential limitations}

We limited our project to modelling, experiments and data processing. The deliverable objective is numerical codes for early detection of autism disorders. This may appear as a limitation, yet our project seems reachable. As a consequence, we should be able to build a PHS for early detection of challenging behaviour in ASD individuals, outside a hospital setting. We will have the possibility to deposit a licence and develop industrial applications.

\section{Author affiliations}

${ }^{1}$ Department of Preventive and Occupational Medicine, University Hospital of Clermont-Ferrand (CHU), Clermont-Ferrand, France

${ }^{2}$ School of Exercise Science, Australian Catholic University, Melbourne, Victoria, Australia

${ }^{3}$ Laboratory of Metabolic Adaptations to Exercise in Physiological and Pathological Conditions, Clermont University, Blaise Pascal University, Aubière, France

${ }^{4}$ Human Nutrition Unit (UNH), Research Centre in Human Nutrition (CRNH), INRA, Clermont-Ferrand, France

${ }^{5}$ Clermont Université, Université Blaise Pascal, Laboratoire de Psychologie Sociale et Cognitive, Clermont-Ferrand, France

${ }^{6}$ CNRS, UMR 6024, LaPSCo, Clermont-Ferrand, France

${ }^{7}$ Signals and Systems Research Unit, National Engineering School of Tunis,

El Manar University, Tunis, Tunisia

${ }^{8}$ Clermont Université, Université Blaise Pascal, Laboratoire de Mathématiques, Clermont-Ferrand, France

${ }^{9}$ CNRS, UMR 6620, LM, Clermont-Ferrand, France

${ }^{10}$ Univ Grenoble Alpes, LPNC, Grenoble, France

${ }^{11}$ CNRS, UMR 5105, LPNC, Grenoble, France

${ }^{12}$ Institut Universitaire de France, Paris, France

Contributors FD was responsible for the ethics committee. $P C$ and $R G$ coordinated the recruitment of patients. $\mathrm{CH}$ and $\mathrm{NK}$ are responsible for data collection. GP is responsible for data processing. PRB, PC and FD are responsible for the overall supervision. FD wrote the first draft. All authors contributed to the study design and read, contributed towards and approved the final manuscript.

Funding The study is integrally funded by the French National Agency for Research (ANR, Agence Nationale de la Recherche), ANR grant number: ANR-12-BS01-0016-01, which is coordinated by PRB.

Competing interests None.

Patient consent Obtained.

Ethics approval Ethical approval has been obtained from Ethics Committee of Clermont-Ferrand (South-East I), France (2014-A00611-46). This exploratory study in an ecological situation received approval from the Ethics Committee of the University Hospital of Clermont-Ferrand. ClinicalTrials identifier NCT02275455.

Provenance and peer review Not commissioned; peer reviewed for ethical and funding approval prior to submission. 
Data sharing statement Original protocol from this study is available by contacting the corresponding author via email.

Open Access This is an Open Access article distributed in accordance with the Creative Commons Attribution Non Commercial (CC BY-NC 4.0) license, which permits others to distribute, remix, adapt, build upon this work non-commercially, and license their derivative works on different terms, provided the original work is properly cited and the use is non-commercial. See: http://creativecommons.org/licenses/by-nc/4.0/

\section{REFERENCES}

1. American Psychiatric Association APADSMTF. Diagnostic and statistical manual of mental disorders: DSM-5. Washington DC: American Psychiatric Association, 2013.

2. Elsabbagh M, Divan G, Koh YJ, et al. Global prevalence of autism and other pervasive developmental disorders. Autism Res 2012;5:160-79.

3. Landrigan PJ. What causes autism? Exploring the environmental contribution. Curr Opin Pediatr 2010;22:219-25.

4. Uddin LQ, Supekar K, Menon V. Reconceptualizing functional brain connectivity in autism from a developmental perspective. Front Hum Neurosci 2013;7:458

5. Leekam SR, Nieto C, Libby SJ, et al. Describing the sensory abnormalities of children and adults with autism. $J$ Autism Dev Disord 2007;37:894-910.

6. Simonoff E, Pickles A, Charman T, et al. Psychiatric disorders in children with autism spectrum disorders: prevalence, comorbidity, and associated factors in a population-derived sample. J Am Acad Child Adolesc Psychiatry 2008;47:921-9.

7. Pfeiffer B, Kinnealey M, Reed C, et al. Sensory modulation and affective disorders in children and adolescents with Asperger's disorder. Am J Occup Ther 2005;59:335-45.

8. Picard RW. Future affective technology for autism and emotion communication. Philos Trans R Soc Lond B Biol Sci 2009;364:3575-84.

9. Dutheil F, Boudet G, Perrier C, et al. JOBSTRESS study: comparison of heart rate variability in emergency physicians working a 24-hour shift or a 14-hour night shift-a randomized trial. Int $J$ Cardiol 2012;158:322-5.

10. Quintana DS, Guastella AJ, Outhred T, et al. Heart rate variability is associated with emotion recognition: direct evidence for a relationship between the autonomic nervous system and social cognition. Int J Psychophysiol 2012;86:168-72.

11. Malliani A, Pagani M, Lombardi $F$, et al. Cardiovascular neural regulation explored in the frequency domain. Circulation 1991;84:482-92.

12. Licht CM, de Geus EJ, van Dyck R, et al. Association between anxiety disorders and heart rate variability in The Netherlands Study of Depression and Anxiety (NESDA). Psychosom Med 2009;71:508-18.

13. Althaus M, Mulder LJ, Mulder G, et al. Cardiac adaptivity to attention-demanding tasks in children with a pervasive developmental disorder not otherwise specified (PDD-NOS). Biol Psychiatry 1999;46:799-809.

14. Ming X, Julu PO, Brimacombe M, et al. Reduced cardiac parasympathetic activity in children with autism. Brain Dev 2005;27:509-16.

15. Storm $\mathrm{H}$. Changes in skin conductance as a tool to monitor nociceptive stimulation and pain. Curr Opin Anaesthesiol 2008;21:796-804.

16. Picard RW, Healey J. Affective wearables. Personal Technol 1997:1:231-40.

17. Kylliäinen A, Hietanen J. Skin conductance responses to another person's gaze in children with autism. J Autism and Dev Disord 2006;36:517-25.

18. Dawson G. Early behavioral intervention, brain plasticity, and the prevention of autism spectrum disorder. Dev Psychopathol 2008;20:775-803.

19. White SW, Oswald D, Ollendick T, et al. Anxiety in children and adolescents with autism spectrum disorders. Clin Psychol Rev 2009;29:216-29.
20. Marco EJ, Hinkley LBN, Hill SS, et al. Sensory processing in autism: a review of neurophysiologic findings. Pediatr Res 2011;69:48R-54R.

21. Wiggins LD, Robins DL, Bakeman R, et al. Brief report: sensory abnormalities as distinguishing symptoms of autism spectrum disorders in young children. J Autism Dev Disord 2009;39: 1087-91.

22. Sigman M, Dissanayake C, Corona R, et al. Social and cardiac responses of young children with autism. Autism 2003;7:205-16.

23. Khalfa N, Bertrand PR, Boudet G, et al. Heart rate regulation processed through wavelet analysis and change detection: some case studies. Acta Biotheoretica 2012;60:109-29.

24. Lord C, Rutter M, Le Couteur A. Autism diagnostic interview-revised: a revised version of a diagnostic interview for caregivers of individuals with possible pervasive developmental disorders. J Autism Dev Disord 1994;24:659-85.

25. Lord C, Risi S, Lambrecht L, et al. The autism diagnostic observation schedule-generic: a standard measure of social and communication deficits associated with the spectrum of autism. J Autism Dev Disord 2000;30:205-23.

26. Sparrow SS, Balla DA, Cicchetti DV. Vineland Adaptive Behavior Scales: Interview Edition, Survey Form Manual. 1984.

27. Simoes RP, Mendes RG, Castello V, et al. Heart-rate variability and blood-lactate threshold interaction during progressive resistance exercise in healthy older men. $J$ Strength Cond Res 2010;24:1313-20.

28. Lewis MJ, Kingsley M, Short AL, et al. Rate of reduction of heart rate variability during exercise as an index of physical work capacity. Scand J Med Sci Sports 2007;17:696-702.

29. Cysarz D, von Bonin D, Brachmann P, et al. Day-to-night time differences in the relationship between cardiorespiratory coordination and heart rate variability. Physiol Meas 2008;29:1281-91.

30. Task Force of the European Society of Cardiology the North American Society of Pacing Electrophysiology. Heart rate variability: standards of measurement, physiological interpretation, and clinical use. Circulation 1996;93:1043-65.

31. Tochikubo O, Ikeda A, Miyajima E, et al. Effects of insufficient sleep on blood pressure monitored by a new multibiomedical recorder. Hypertension 1996;27:1318-24.

32. Mallat S. A wavelet tour of signal processing. Boston: Academic Press, 2009.

33. Ayache A, Bertrand Pierre R. Discretization error of wavelet coefficient for fractal like processes. Adv Pure Appl Math 2011;2:297-321.

34. Basseville M, Nikiforov IV. Detection of abrupt changes: theory and application. Englewood cliffs, NJ: Prentice Hall, 1993:469.

35. Bertrand PR, Fhima M, Guillin A. Off-line detection of multiple change points by the filtered derivative with $p$-value method. Sequential Anal 2011;30:172-207.

36. Bardet JM, Bertrand PR. A non-parametric estimator of the spectral density of a continuous-time Gaussian process observed at random times. Scand J Stat 2010;37:458-76.

37. Mandelbrot BB, Van Ness JW. Fractional Brownian motions, fractional noises and applications. SIAM Rev 1968;10:422-37.

38. Bardet JM, Surgailis D. Measuring the roughness of random paths by increment ratios. Bernoulli 2011:17:507-826.

39. Kaufman L, Rousseeuw PJ. Finding groups in data: an introduction to cluster analysis: John Wiley \& Sons, Inc, 2008.

40. Azzaoui N, Guillin A, Dutheil F, et al. Classifying heartrate by change detection and wavelet methods for emergency physicians. ESAIM Proceedings; 2014.

41. Hjortskov N, Rissen D, Blangsted AK, et al. The effect of mental stress on heart rate variability and blood pressure during computer work. Eur J Appl Physiol 2004;92:84-9.

42. Mandelbrot BB. The variation of certain speculative prices. J Bus 1963;36:394-419.

43. Engle RF, Granger CW. Autoregressive conditional heteroscedasticity with estimates of the variance of United Kingdom Inflation. Econometrica 1982;50:987-1008.

44. Granger CW. Time series analysis, cointegration, and applications. Am Econ Rev 2004;94:421-5.

45. Hansen LP. Large sample properties of generalized methods of moments estimators. Econometrica 1982;50:1029-54. 